\title{
Fullerene Carbon Soot Characterization for Application as a New Solar Energy Absorption Material
}

Luis Bernardo López-Sosa ${ }^{1}$, Francisco Robles-Hernandez ${ }^{2}$, Mauricio González-Avilés ${ }^{3}$, Ismael SantosRamos $^{1}$, J. Zárate-Medina ${ }^{1}$, Luis Mariano Hernández-Ramírez ${ }^{1}$ and Ariosto Medina-Flores ${ }^{1}$

${ }^{1}$ Universidad Michoacana de San Nicolás de Hidalgo, Morelia, Michoacan de Ocampo, Mexico, ${ }^{2}$ University of Houston, Houston, Texas, United States, ${ }^{3}$ Universidad Intercultural Indígena de Michoacán, Pátzcuaro, Michoacan de Ocampo, Mexico

Solar coatings not only contribute to the efficiency of solar thermal technologies, they also significantly influence the cost and environmental impact of the entire device [1]. These coatings should also consider that the absorption of light by a material depends on its electronic structure, the wavelength of the light and the materials that are solid, that is, any characteristic of the surface, such as wells, hollows or peaks. [2. 3]. The application of these materials generates various coatings that have been studied for a long time, and can be specifically classified into [4-5]: (a) textured metal surfaces (b) intrinsic selective materials (c) multilayer absorbers (d) metals with dielectric compounds, (e) selective solar transmission coating on a black body type absorbent and (f) absorbent paints with organic materials. Some materials have been developed to generate optical and structural properties that are highly absorbed in the solar spectrum (300$2500 \mathrm{~nm}$ ). Particularly, this proposal suggests the characterization of fullerene ashes, obtained by the Krätschmer method [6], as a new material for absorbing solar energy; This material has been studied to generate new carbon allotropes with some characteristics similar to graphene [6]. However, little has been studied of the optical properties of this material, so this research is interesting

The characterization of fullerene ashes (FCS) was performed using X-ray Diffraction (DRX), using a D8 Adavance Davinci diffraction system. Scanning Electron Microscopy (SEM) was used, using a Model Jeol JSM 7600F field emission equipment. Also, Raman Spectroscopy was used, through a Bruker Raman Senterra device. For infrared spectroscopy a Bruker tensor model 27 (FTIR) was used. And for UV-VISNIR Spectroscopy, a Cary 5000 device with integrating sphere was used.

Fig. 1 (a) shows the XRD spectra of FCS, which is essentially amorphous except for some traces of fullerene; therefore, the reflections are not very clear in the background [7]. The prominent reflection at approximately 25.4 is the result of ordered short-range carbon structures. These structures correspond to a distribution of graphene scales with a small lateral size (almost clusters), stacked without tightening at variable distances higher than those of graphite. Due to the absence of other reflections typical of graphite, it is proposed that this soot have a limited arrangement in the "c" direction [7]; This is a structured or quasi amorphous material of short range. It can be concluded that our soot does not have a significant fullerene presence and is practically amorphous. Regarding the SEM characterization (Fig. 1b), the presence of nanometric particles for the SCF can be seen. The agglomerates show nanometric sizes, that is, the smaller particles will have sizes smaller than $50 \mathrm{~nm}$. Fig. 1 (c) shows the results of the FTIR spectroscopy. The bands show the correspondence of the FCS's fulerene structures, bands that match those reported by the specialty literature. At $1182 \mathrm{~cm}-1$ and $1430 \mathrm{~cm}-1$, the characteristic bands of the pristine C60, a defined fullerene structure, are reported. While at $1720 \mathrm{~cm}-1$ it is associated with the presence of the carboxyl group; and the remaining bands at 1159 1307, 1550, 1649 and $1784 \mathrm{~cm}-1$, show the possible existence of other types of fulerenes, perhaps of type C70 [8]

On the other hand, by Raman spectroscopy, fig. 1 (d), the presence of characteristic bands for carbon allotropes can be seen. The D band $(1350 \mathrm{~cm}-1)$ that shows the amorphous or disordered graphite 
characteristics, while the $\mathrm{G}$ band $(1580 \mathrm{~cm}-1)$ shows the first order dispersion of the graphite (crystalline) plane, that is, the $\mathrm{sp}^{2}$ hybridized domain of the material [9]. Likewise, a very thin third band can be seen at approximately $2750 \mathrm{~cm}-1$, known as the 2D band; which has been reported by Xie et al., Singh et al. and Akhavan [9], which is attributed to the appearance of few layers of graphene in carbonaceous materials, which can generate higher value bands, corresponding to multilayer graphene. The presence of $\mathrm{sp}^{2}$ bonds, in carbonaceous materials, is usually attributed to the light absorption capacity. On the other hand, by means of UV-VIS-NIR characterization, it was possible to evaluate the absorption capacity of the FCS. It has been evaluated throughout the solar spectrum, using the 1.5 A.M. Fig. 1 (e) shows the results of this analysis. It is appreciated that throughout the solar spectrum the reflectance of the FCS is high, which indicates that it has a high capacity for absorbing solar energy. It has also been evaluated, a coating of FCS in comparison with the performance of commercial paint for high temperature (Comex $\AA$, HT Paint). In the graph of UV-VIS-NIR it can be observed that this painting reflects a lot from $700 \mathrm{~nm}$, therefore the infrared is not absorbed.

The absorption capacity is probably due to the domain of $\mathrm{sp}^{2}$ bonds, present in this material. Although to clarify, it is necessary to carry out complementary analyzes that contribute to support this statement. In future work, the analysis will be shown by X-ray Photoemitted Electron Spectroscopy (XPS), as well as the thermal characterization of the operation of this material.
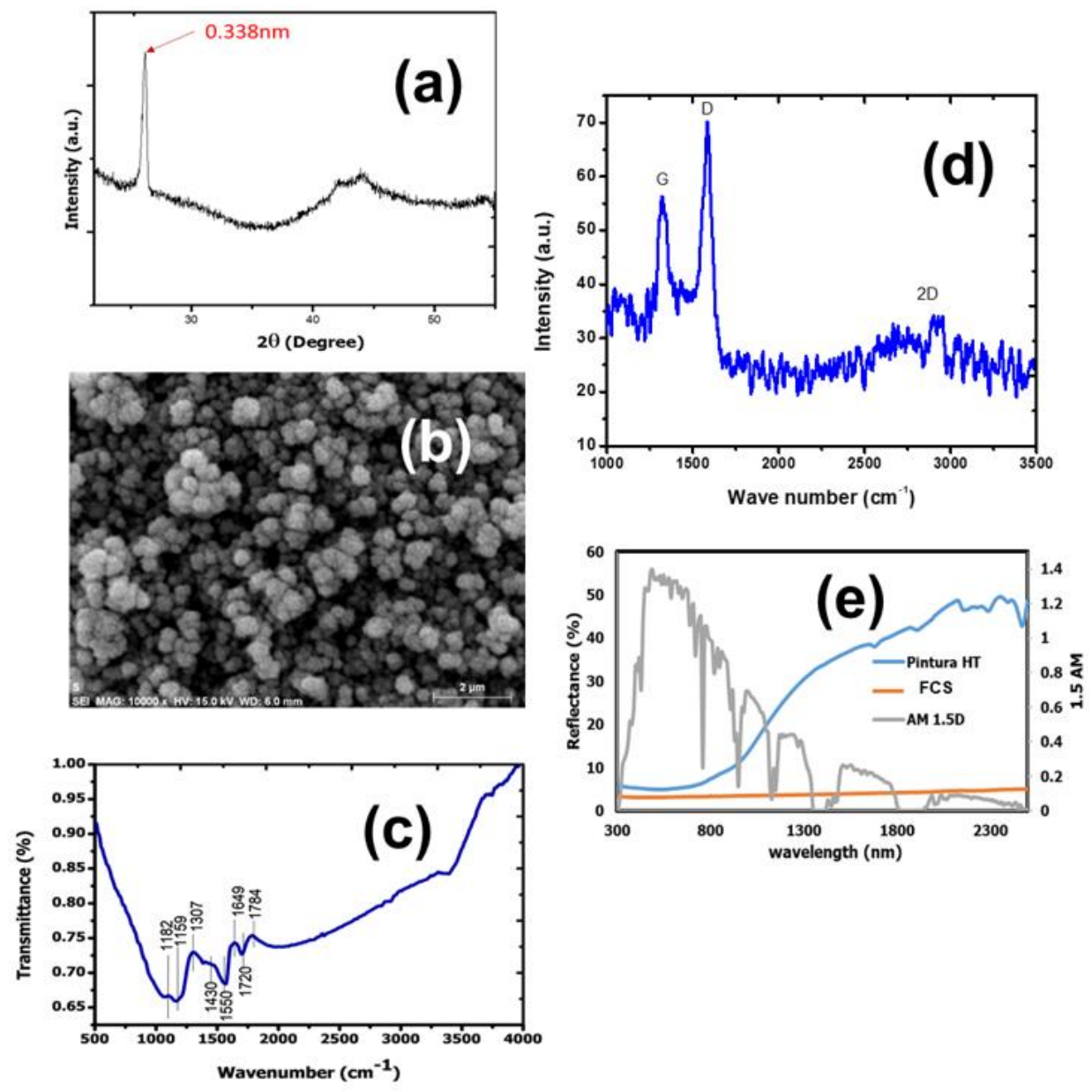

Figure 1. Characterization: (a) DRX (b) SEM (c) FTIR (d) Raman and (e) UV-VIS-NIR 


\section{References}

[1] Altun-Çiftçioğlu, Gökulu, F. Kadırgan \& M. Kadırgan. Life cycle assessment (LCA) of a solar selective surface produced by continuous process and solar flat collectors. Solar Energy, 135, 284-290. 2016

[2] P. Feynman. QED The strange theory of light and matter. 1st ed. Princeton: Princeton University Press; 1985.

[3] Granqvist. Solar energy materials. Applied Physics A, 52(2), 83-93. 1991.

[4] E. Kennedy. Review of mid-to high-temperature solar selective absorber materials(No. NREL/TP520-31267). National Renewable Energy Lab., Golden, CO.(US). 2002

[5] Atkinson, C. Sansom, H. Almond \& C. Shaw. Coatings for concentrating solar systems-A review. Renewable and Sustainable Energy Reviews, 45, 113-122. 2015.

[6] Calderon, I Estrada-Guel, I., Alvarez-Ramírez, F., Hadjiev, V. G., \& Hernandez, F. R. Morphed graphene nanostructures: experimental evidence for existence. Carbon, 102, 288-296. 2016.

[7] Fals, V. Hadjiev \& F. Hernández. Multi-functional fullerene soot/alumina composites with improved toughness and electrical conductivity. Materials Science and Engineering: A, 558, 13-20. 2012.

[8] Andrievsky, V. Klochkov, A.Bordyuh \& G. Dovbeshko. Comparative analysis of two aqueouscolloidal solutions of $\mathrm{C} 60$ fullerene with help of FTIR reflectance and UV-Vis spectroscopy. Chemical Physics Letters, 364(1-2), 8-17. 2002.

[9] Singh, D. Singh, S. Singh \& A. Pandey. Candle soot derived carbon nanoparticles: Assessment of physico-chemical properties, cytotoxicity and genotoxicity. Chemosphere, 214, 130-135. 2019 